Trauma Berufskrankh 2005 · 7[Suppl 1]:S39-S44 DOI 10.1007/s10039-004-0954-x

Online publiziert: 5. November 2004

(c) Springer Medizin Verlag 2004

S. Fuchs ${ }^{1} \cdot$ S. Wallstabe ${ }^{1} \cdot$ M. E. Wenzl $\left.\right|^{2} \cdot$ Ch. Jürgens $^{1} \cdot$ D. Wolter ${ }^{1}$

${ }^{1}$ Abteilung für Unfall- und Wiederherstellungschirurgie,

BG-Unfallkrankenhaus, Hamburg

${ }^{2}$ Abteilung für Unfall- und Wiederherstellungschirurgie,

Universitätsklinikum Schleswig-Holstein, Campus Lübeck

\title{
Osteosynthese proximaler Tibiafrakturen
}

\section{Die Bedeutung der multidirektionalen Winkelstabilität}

Weitere Schwierigkeiten ergeben sich durch kurze, proximale Fragmente mit Ansatzpunkten von ligamentären Strukturen, deren Zugrichtung sowohl nach proximal als auch nach distal gerichtet sind. Bei fehlender knöcherner metaphysärer Abstützung kann es zu sekundären Stellungsverlusten bei Osteosynthesen mit ungenügender Stabilität kommen.

Die klassische Marknagelosteosynthese kann zu Fehlstellungen und Instabilitäten führen, insbesondere bei sehr proximalen Frakturen.

Hier bieten sich winkelstabile Fixateur-interne-Systeme an. Dabei sind die multidirektionalen winkelstabilen Systeme leistungsfähiger als die unidirektionalen, welche auf den Franzosen Paul Rein- hold (1931) zurückgehen [7, 16]. Bei kurzen Fragmenten ist bei nicht winkelstabilen Plattensystemen die Auflagefläche der Platte zu klein, um eine suffiziente Abstützung zu erreichen, sodass es zu einem Versagen der Osteosynthese kommen kann. Bei winkelstabilen Fixateur-interne-Systemen wird die Kraft auf alle Abschnitte der winkelstabilen Schrauben übertragen, sodass eine gleichmäßigere Verteilung derselben über den gesamten Knochenquerschnitt und eine Vergrößerung der Auflagefläche resultieren [16]. Die Winkelstabilität verhindert zusätzlich das Heraustreten der Schrauben. Untersuchungen konnten einen Stabilitätsgewinn einer multidirektionalen winkelstabilen Osteosynthese gegenüber einer konventionellen Plattenosder Fälle.
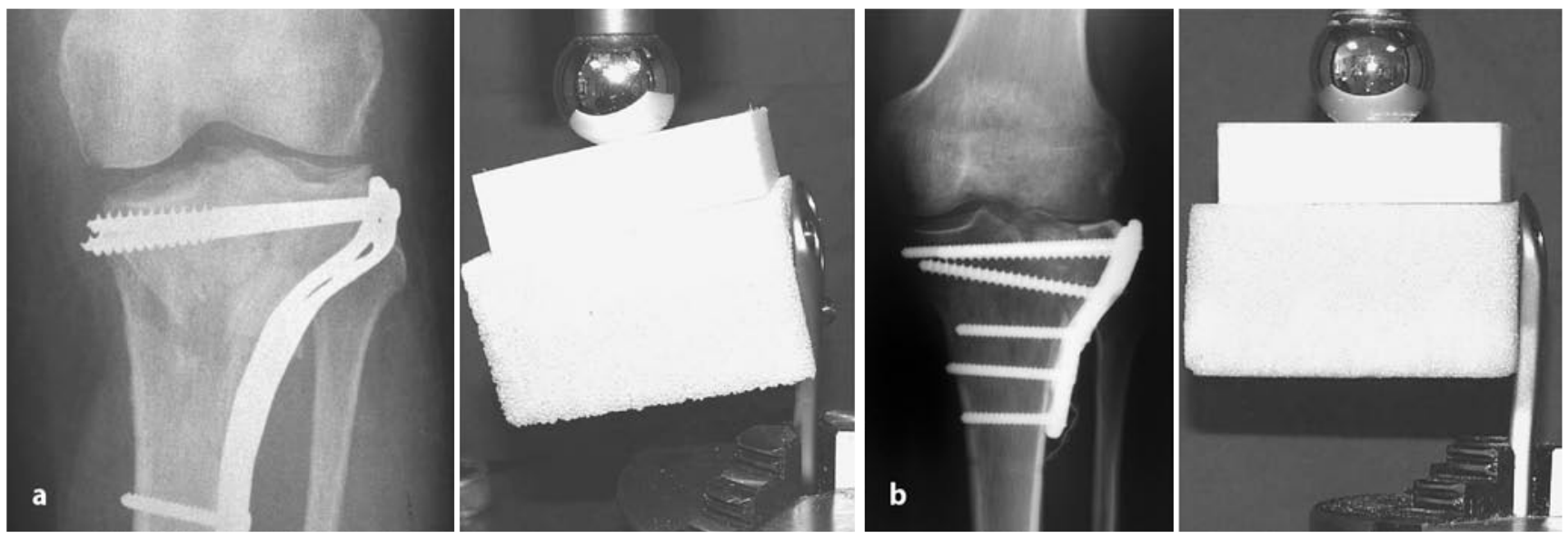

Abb. $1 \Delta$ Simulation einer metaphysären Plattenosteosynthese, a Versagen der nicht winkelstabilen, b Stabilität der winkelstabilen Schrauben-Platten-Verbindung (axiale Last $700 \mathrm{~N}$, Biegelast $28 \mathrm{Nm}$ ) 


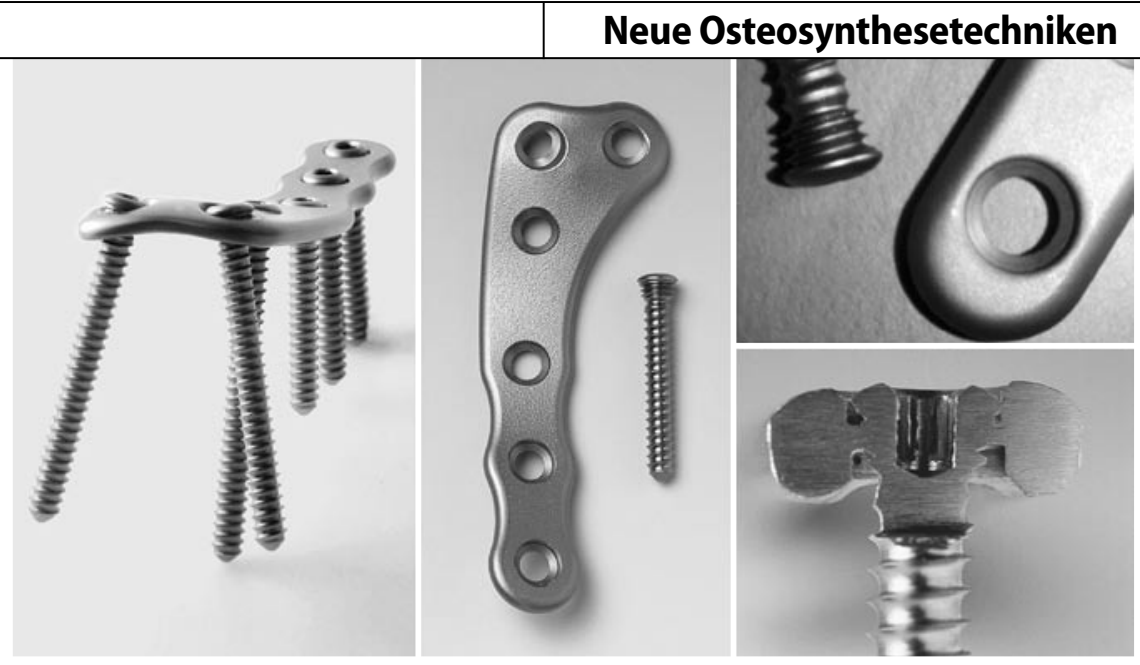

Abb. $2 \Delta$ Titanfixateur interne (TiFix) für die proximale Tibia, multidirektionale Winkelstabilität mit Lippenkonstruktion

teosynthese sowohl im Bereich der Femurkondylen als auch des Tibiakopfs um den Faktor 3,1 nachweisen (• Abb. 1) [9].

Die Winkelstabilität ermöglicht Osteosynthesen im Sinne von minimalinvasiven Operationstechniken in Untertunnelungstechnik und Einschieben der Implantate, sodass der Weichteilsituation des Tibiakopfs und der Weichteilschädigung nach Trauma Rechnung getragen werden kann.

\section{Material und Methode}

Der multidirektionale winkelstabile Titanfixateur interne für die proximale Tibia wurde von Januar 1999-Dezember 2003 bei insgesamt 42 Patienten eingesetzt. Es handelte es sich um 33 Männer und 9 Frauen mit einem Durchschnittsalter von 45,4 Jahren (17-86 Jahre).

Das Design des Titanfixateur interne (Tifix, Litos Hamburg) weist ein LowKontakt-Prinzip auf und ist der anatomischen Form des lateralen bzw. medialen Tibiakopfs angepasst (• Abb. 2). Die Winkelstabilität wird durch die Gewindeverbindung des Schraubenkopfs aus hartem Reintitan Grad 4 und der speziellen Lippenkonstruktion des Plattenlochs aus weichem Reintitan Grad o realisiert, wobei sich der Schraubenkopf das Gewinde selbst formt. Die Schraube kann multidirektional in Winkeln bis $\mathrm{zu} 30^{\circ}$ eingebracht werden ( $\bullet$ Abb. 2) $[3,9,14]$.

Die Indikationen für den Einsatz des Titanfixateur interne an der proximalen $\mathrm{Ti}$ bia stellten bei 23 Patienten Frakturen, bei 13 Pseudarthrosen dar. In 6 Fällen wurde eine Umstellungsosteotomie mit einem winkelstabilen Titanfixateur interne stabilisiert.

Das Therapiekonzept der Frakturversorgung sieht 2 Möglichkeiten vor:

- Bei akzeptablen Hautweichteilverhältnissen wird eine primäre Stabilisierung mit dem Titanfixateur interne innerhalb der ersten $6 \mathrm{~h}$ angestrebt.

- Bei komplexen Weichteilsituationen sowie offenen Frakturen wird zunächst ein Fixateur externe zur Stabilisierung der Fraktur Gelenk übergreifend angelegt und entsprechend der Weichteilkonsolidierung ein sekundärer Umstieg durchgeführt.

In 11 Fällen war eine primäre Stabilisierung der Fraktur möglich. In weiteren 11 Fällen erfolgte ein Umstieg von einem $\mathrm{Fi}$ xateur externe nach einer durchschnittlichen Liegezeit von 26,8 Tagen (7-80 Tage). Die Anlage des Fixateur externe wurde so vorgenommen, dass das spätere Implantatlager des Fixateur interne nicht tangiert wurde. Zusätzlich konnte vor dem Zweiteingriff mit dem Hexapodensystem eine exaktere Reposition der Fraktur ohne Narkose vorgenommen werden. In 1 Fall erfolgte ein sekundärer Umstieg auf den Titanfixateur interne nach primärer Plattenosteosynthese, da sich kurzfristig ein sekundärer Korrekturverlust abzeichnete.

Als Begleitverletzung bei den Frakturen sahen wir in 2 Fällen eine Gefäßverletzung der A. poplitea (• Abb. 3), in 4 Fällen einen N.-peronaeus-Schaden sowie in
2 Fällen ein Kompartmentsyndrom. Die Gefäßverletzungen wurden bei primärer Frakturstabilisierung im Fixateur externe mit einem Veneninterponat rekonstruiert. Die Kompartmentsyndrome erforderten eine Fasziotomie.

Den 13 Pseudarthrosen waren in 5 Fällen Vorbehandlungen mit einer Plattenosteosynthese, in 5 Fällen mit Fixateur-externe-Systemen sowie in 3 Fällen mit UTN vorausgegangen. Die Pseudarthrosen bestanden im Durchschnitt 15,6 Monate (642 Monate) mit durchschnittlich 1,4 Voroperationen (1-3). In allen Fällen erfolgte eine autologe Spongiosaplastik.

Bei 6 Patienten wurde eine Umstellungsosteotomie bei konsolidierter Fehlstellung mittels Titanfixateur interne korrigiert.

Der Titanfixateur interne konnte in 34 Fällen $(80,9 \%)$ minimalinvasiv eingebracht werden.

\section{Ergebnisse}

Zum Untersuchungszeitpunkt im März 2004 waren die Behandlungen bei 39 Patienten (92,85\%) abgeschlossen. Es fand sich in allen Fällen eine knöcherne Konsolidierung der Fraktur bzw. Pseudarthrose oder Korrekturosteotomie.

In 1 Fall war eine 2. autologe Spongiosaplastik bei einer langstreckigen proximalen Tibiadefektfraktur mit verzögerter Knochenheilung notwendig (• Abb. 4).

Ein Implantatversagen oder ein sekundärer Korrekturverlust fanden sich in keinem Fall. In 1 Fall resultierte eine Achsfehlstellung bei primärer Kettenverletzung der unteren Extremität, sodass die primäre Ausrichtung der Beinachse erschwert war.

Ein Plattenlagerinfekt bei positivem Keimnachweis zum Zeitpunkt der Implantation des Titanfixateur interne bei Pseudarthrosenrevision führte zu einer vorzeitigen Materialentfernung bei gesicherter knöcherner Konsolidierung. Ein sekundär auftretendes Hämatom am Tibiakopf musste ausgeräumt werden. Von den primär 4 bestandenen N.-peronaeusSchäden waren 3 vollständig rückläufig, bei 1 Patienten fand sich ein Restdefizit.

An allgemeinen Komplikationen wurden 1 Oberschenkelthrombose (• Abb. 5) und 3 ipsilaterale Unterschenkelthrombosen gesehen. 


\section{Diskussion}

Die proximale Tibiafraktur stellt weiterhin eine Herausforderung dar. Die klassische intramedulläre Markraumschienung gilt bei geschlossenen Frakturen des diaphysären Bereichs als die Methode der Wahl. Bei proximalen Frakturen wurden nach Nagelosteosynthesen postoperativ vermehrt Fehlstellungen und verbleibende bzw. sekundär auftretende Instabilitäten nachgewiesen. Nach intramedullärer Osteosynthese von Tibiaschaftfrakturen fanden Freedmann u. Johnson [2] Achsabweichungen von $12 \%$. Differenziert nach den Lokalisationen betrugen diese bei Frakturen des proximalen Drittels $58 \%$. In der Literatur wurden Achsfehlstellungen in 4484\% [12] der proximalen Tibiaschaftfrakturen angegeben. In der Regel fanden sich in absteigender Häufigkeit Valgusfehlstellungen, Antekurvationsfehlstellungen und Varusfehlstellungen. Sie resultierten nach Analysen von Freedmann u. Johnson [2] und Henley et al. [4] zum einen aus den Repositionsergebnissen, die bei proximalen Tibiafrakturen aufgrund des Muskelzugs am proximalen Fragment zur Dorsal- und Valgusabkippung neigen, zum anderen aus einer inkorrekten Nageleintrittstelle. Abweichungen hierbei führen $\mathrm{zu}$ Valgus- bzw. Varusfehlstellungen des proximalen Fragments.

Aufgrund der hohen mechanischen Beanspruchung besteht die Gefahr einer postoperativ verbleibenden oder auch sekundär auftretenden Instabilität des Knochen-Implantat-Verbunds, da eine Führung des Nagels durch die Kortikalis im trichterförmig zulaufenden Tibiakopf nicht vorhanden ist. Lang et al. [6] berichteten über $25 \%$ solcher Instabilitäten, wobei in der Mehrzahl ihrer Fälle proximal nur eine Verrieglungsschraube zur Anwendung kam.

Die mechanische Beanspruchung des Knochen-Implantat-Verbunds nimmt mit der Frakturhöhe zu, während die durch den Nagel gewährleistete Stabilität aufgrund des fehlenden Kortikaliskontakts im Tibiakopf abnimmt. Des Weiteren hat die Qualität des Knochens einen wesentlichen Einfluss auf die Stabilität der Osteosynthese. Hier kommen die Vorteile des winkelstabilen Fixateur-interne-Systems besonders zum Tragen.

Trauma Berufskrankh $2005 \cdot 7[$ Suppl 1]:S39-S44

DOI 10.1007/s10039-004-0954-x

c) Springer Medizin Verlag 2004

S. Fuchs · S. Wallstabe · M. E. Wenzl · Ch. Jürgens · D. Wolter

\section{Osteosynthese proximaler Tibiafrakturen. Die Bedeutung der multidirektionalen Winkelstabilität}

\section{Zusammenfassung}

Von Januar 1999-Dezember 2003 wurde der multidirektionale winkelstabile Titanfixateur interne (TiFix) für die proximale Tibia bei 42 Patienten eingesetzt. Bei $23 \mathrm{~Pa}$ tienten wurden Frakturen stabilisiert, dabei fand in 11 Fällen ein Umstieg vom Fixateur externe, in 1 Fall von einer konventionellen Platte statt. 11 Frakturen wurden primär versorgt. Des Weiteren wurden 13 Patienten mit Pseudarthrose sowie 6 Patienten mit Fehlstellungen therapiert. In 34 Fällen $(80,9 \%)$ wurde das Implantat minimalinvasiv eingebracht. Zum Nachuntersuchungszeitpunkt waren 39 Behandlungen (92,9\%) abgeschlossen. In allen Fällen konnte eine vollständige Durchbauung erreicht werden. Ein Implantatversagen zeigte sich nicht. Postoperativ fand sich ein Plattenla-

\section{Osteosynthesis of fractures of the proximal tibia. Significance of the multidirectional angular stability}

\section{Abstract}

From January 1999 until December 2003 we used a plate fixator with multidirectional blocked screws (TiFix) for internal fixation of the proximal tibia in 42 patients. In 23 cases this was used to stabilise fractures: in 11 cases for primary fracture management, in a further 11 after initial management with an external fixator and in 1 after initial fixation with a conventional plate. We also used the TiFix plate in 13 operations for pseudarthrosis and 6 for axis deviation after bone healing. In 34 (80.9\%) of the total of 42 cases it was possible to implant the plate in a minimally invasive procedure. At the time of clinical follow up the treatment was complete in 39 (92.9\%) cases. Fracture consolidation has been achieved in all cases; no implant failures have been observed. One patient gerinfekt bei einer Pseudarthrose, bei der ein Staphylokokkenbefall zum Zeitpunkt des Verfahrenswechsels nachgewiesen werden konnte. Die Ergebnisse unterstreichen die Leistungsfähigkeit des multidirektionalen, winkelstabilen Fixateur-interneSystems bei der Versorgung proximaler Tibiafrakturen. In Verbindung mit minimalinvasiven Operationstechniken stellt diese Osteosyntheseform für uns erste Priorität im Bereich der proximalen Tibia dar.

\section{Schlüsselwörter}

Proximale Tibiafraktur .

Multidirektionale Winkelstabilität .

Fixateur interne - Minimalinvasive

Operationstechnik · Tifix developed a deep wound infection around the plate after revision for pseudarthrosis; in this patient swabs taken intraoperatively had proved positive for staphylococci. Our results underline the high effectiveness of this internal fixator system with multidirectional angular stability in osteosynthesis for proximal tibial fractures. This implant together with the minimally invasive operation technique achieves superior results and low morbidity in the primary treatment of proximal tibial fractures and in treatment of nonunion after such fractures sustained earlier.

\section{Keywords}

Proximal tibial fracture $\cdot$ Multidirectional angular stability · Internal fixator ·

Minimally invasive osteosynthesis · Tifix 

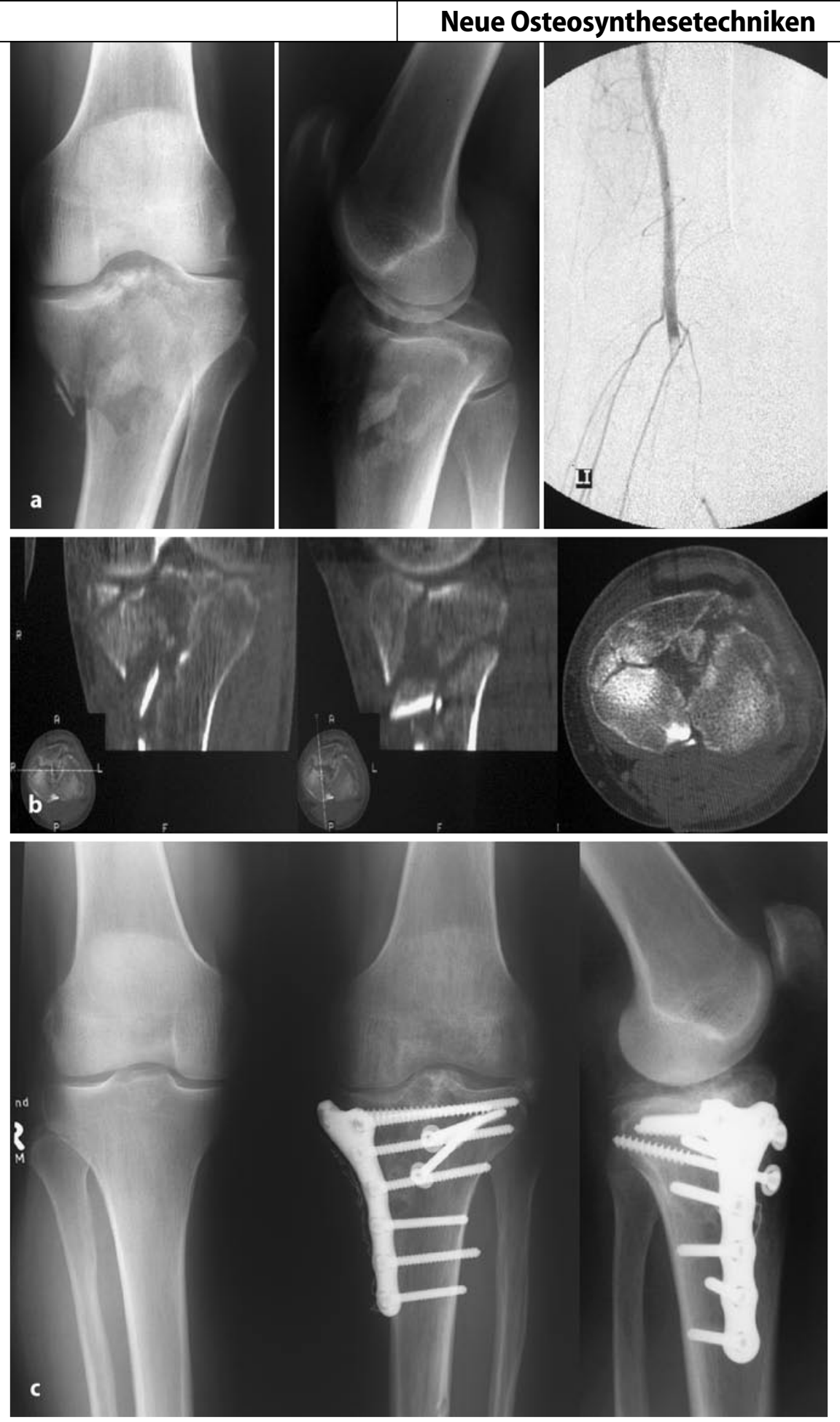

Abb. $3 \triangle$ 44-jähriger Patient mit Tibiakopffraktur und Intimazerreißung der A. poplitea nach hinterer Knieluxation, a Unfallbilder mit Angiogramm, b CT nach Primärstabilisierung im Fixateur externe mit Rekonstruktion der Arterie, c postoperatives Röntgenbild 12 Monate nach Versorgung

Man unterscheidet zwischen den unidirektionalen Systemen nach Paul Reinhold [7] und den multidirektionalen winkelstabilen Plattensystemen [16]. Letztere haben zahlreiche Vorteile für den Operateur und den Patienten [16]. Wir verwenden daher nur Platten mit dieser Technologie.
Bei der winkelstabilen Osteosynthese ist die Stabilität nicht abhängig von der Anzugskraft der Schrauben im Knochen, da sie nicht, wie bei der konventionellen Plattenosteosynthese, durch den Reibschluss der Platte mit dem Knochen, sondern nach dem Prinzip des eingespannten $\mathrm{He}$ - bels erreicht wird. Durch die Verriegelung der Schraubenköpfe in der Platte können die Schrauben nicht gegen diese verkippen.

Die einwirkende Kraft wird großflächig auf alle Abschnitte der winkelstabilen Schrauben übertragen. Daraus resultieren eine Vergrößerung der Auflagefläche und damit eine gleichmäßigere Kraftverteilung über den gesamten Knochenquerschnitt [9]. Hieraus ergeben sich insbesondere im spongiösen, metaphysären Bereich, bei Osteoporose und bei kurzen Fragmenten Vorteile. Die Winkelstabilität gewährleistet eine hohe Primärstabilität der Osteosynthese [9]. Der prekären Weichteilsituation am proximalen Tibiaabschnitt kann durch weichteilschonende Operationstechniken im Sinn von minimalinvasiven Zugängen unter Schonung der peri- und enostalen Vaskularisation des Knochens Rechnung getragen werden.

Die propagierte bilaterale Plattenosteosynthese [5], die eine erhöhte Stabilität erbringt, aber auch zu vermehrten Weichteilproblemen und tiefen Infektionen führen kann, ist beim Einsatz winkelstabiler Osteosynthesesysteme nicht notwendig. Biomechanische Untersuchungen [13] an Leichentibiae konnten bei lateraler Plattenpositionierung eines winkelstabilen Plattenfixateur interne eine signifikant höhere Stabilität aufzeigen als bei konventioneller Abstützplattenosteosynthese, sodass in der Regel eine unilaterale winkelstabile Osteosynthese als ausreichend angesehen werden kann.

Aufgrund der dargestellten Ergebnisse streben wir zunächst bei akzeptablen Hautweichteilverhältnissen eine primäre Stabilisierung der proximalen Tibiafrakturen mit einem winkelstabilen Implantat an. Bei offenen Frakturen oder Frakturen mit erheblicher Weichteilkontusion wird zunächst die Fixateur-externe-Stabilisierung als Mittel der Wahl durchgeführt. Im Anschluss kann dann entsprechend der Weichteilsituation und bei reizfreien Pineintrittsstellen ein Verfahrenswechsel auf den Titanfixateur interne erfolgen.

Aufgrund der bislang erzielten Ergebnisse mit einem winkelstabilen Fixateur-interne-System im Bereich der proximalen Tibia kann dieses Verfahren als eine sinnvolle und leistungsfähige Therapiemöglich- 

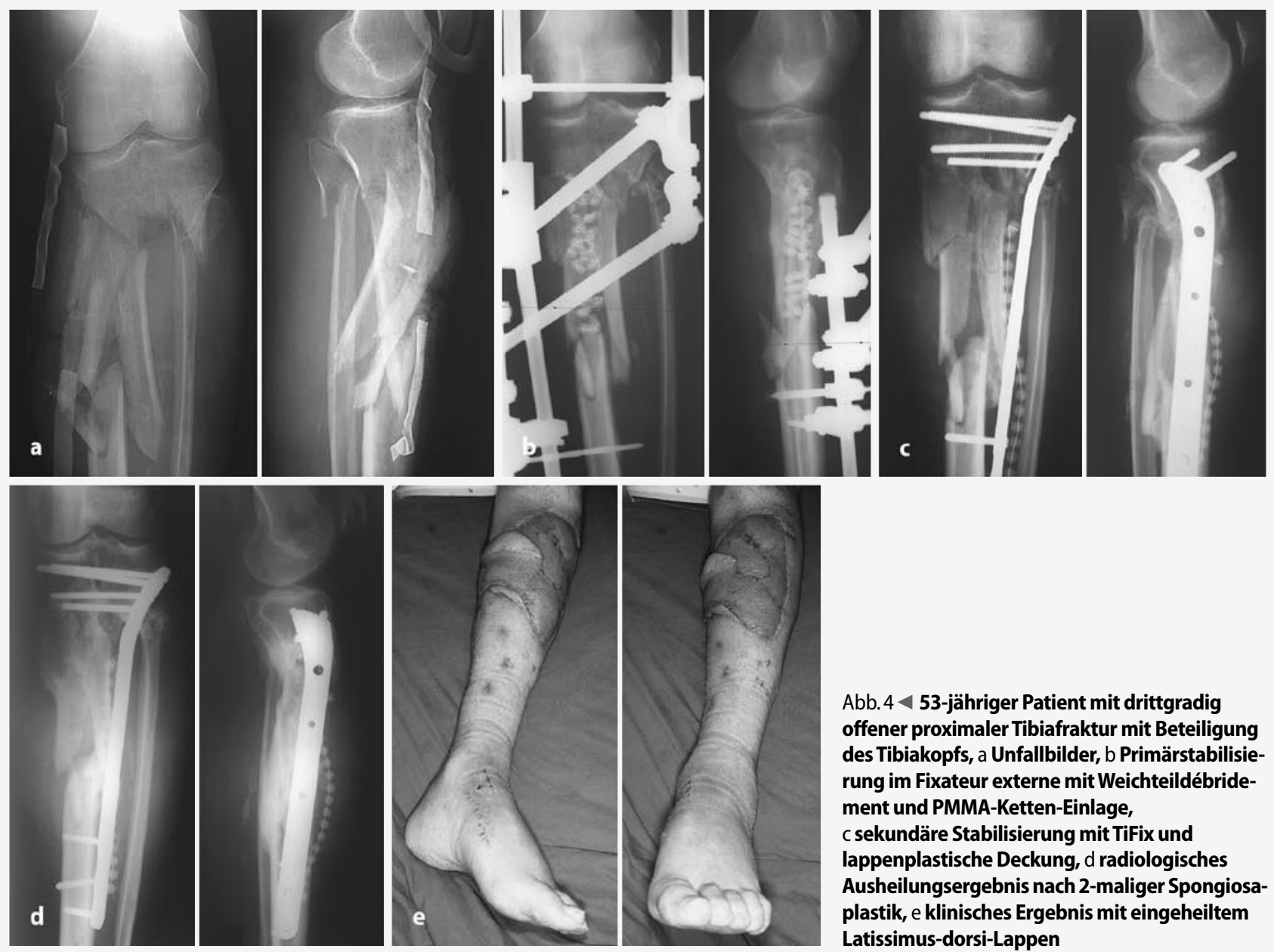

Abb. $4<$ 53-jähriger Patient mit drittgradig offener proximaler Tibiafraktur mit Beteiligung des Tibiakopfs, a Unfallbilder, b Primärstabilisierung im Fixateur externe mit Weichteildébridement und PMMA-Ketten-Einlage,

C sekundäre Stabilisierung mit TiFix und lappenplastische Deckung, $d$ radiologisches Ausheilungsergebnis nach 2-maliger Spongiosaplastik, e klinisches Ergebnis mit eingeheiltem Latissimus-dorsi-Lappen
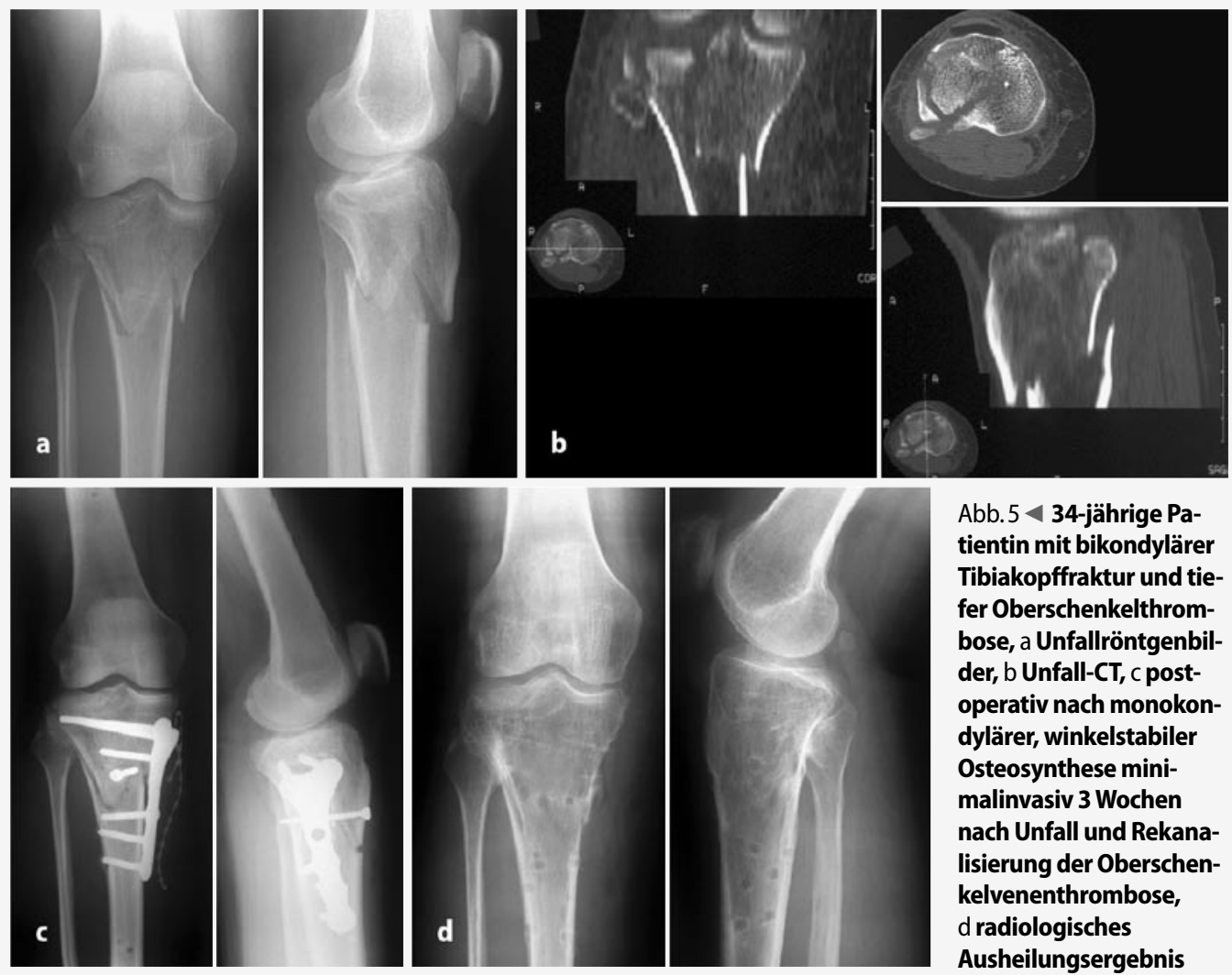

Abb. $5<$ 34-jährige Pa-

tientin mit bikondylärer

Tibiakopffraktur und tiefer Oberschenkelthrom-

bose, a Unfallröntgenbilder, $b$ Unfall-CT, $c$ postoperativ nach monokondylärer, winkelstabiler Osteosynthese minimalinvasiv 3 Wochen nach Unfall und Rekanalisierung der Oberschenkelvenenthrombose, $\mathrm{d}$ radiologisches Ausheilungsergebnis 
keit für die Behandlung der proximalen Tibiafrakturen angesehen werden.

Die Verbindung der multidirektionalen Winkelstabilität mit minimalinvasiven Operationstechniken stellt u. E. das Behandlungskonzept erster Priorität für proximale Tibiafrakturen dar.

\section{Korrespondierender Autor}

\section{Dr. S. Fuchs}

Abteilung für Unfall- und Wiederherstellungschirurgie, BG-Unfallkrankenhaus, Bergedorfer Straße 10, 21033 Hamburg E-Mail:S.Fuchs@buk-hamburg.de

Interessenkonflikt: Der korrespondierende Autor versichert, dass keine Verbindungen mit einer Firma, deren Produkt in dem Artikel genannt ist, oder einer Firma, die ein Konkurrenzprodukt vertreibt, bestehen.

\section{Literatur}

1. Ahlers J, Issendorf WD von (1992) Häufigkeit und Ursachen von Fehlstellungen nach Unterschenkelmarknagelungen. Unfallchirurgie 18: 31

2. Freedmann EL, Johnson EE (1995) Radiographic analysis of tibial fracture malalignment following intramedullary nailing. Clin Orthop 315: 25

3. Fuchs S, Wolter D, Kranz H-W et al. (2001) Titan-Fixateur-interne-Systeme mit multidrektionaler Winkelstabilität im Unterschenkel- und Fußbereich. Trauma Berufskrankh 3 [Suppl 4]: S447-S453

4. Henley MB, Meier M, Tencer AF (1993) Influences of some design parameters on the biomechanics of the unreamed tibial intramedullary nail. J Orthop Trauma 7: 311

5. Horwitz DS, Bachus KN, Craig MA et al. (1999) A biomechanical analysis of internal fixation of complex tibial plateau fractures. J Orthop Trauma 8: 545-549

6. Lang GJ, Cohen BE, Bosse MJ et al. (1995) Proximal third tibial shaft fractures. Should they be nailed? Clin Orthop 315: 64

7. Reinhold MP, Collin et Cie (1931) Système de cramponage aux os des attelles d'osteosynthese et de tout système de maintien des fractures. Brevet Invent 742.618, République Francaise

8. Sarmiento A, Gersten LM, Sobol PA et al. (1989) Tibial shaft fractures treated with functional braces. Experience with 780 fractures. J Bone Joint Surg $\mathrm{Br}$ 71:602

9. Seide K, Morlock MM, Schümann U et al. (1999) Wirkprinzipien der winkelstabilen Platten-Schrauben-Verbindung bei Fixateur-interne-Osteosynthesen. Trauma Berufskrankh 1:320-325

10. Seide K, Jürgens Ch, Wenzl M et al. (2003) Achskorrekturen und Pseudarthrosenbehandlung nach Unterschenkelfrakturen durch minimalinvasiven $\mathrm{Zu}$ gang und Fixateur interne. Trauma Berufskrankh 5 [Suppl 1]: S67-S72

11. Tytherleigh-Strong GM, Keating JF, Court-Brown CM (1997) Extra-articular fractures of the proximal tibial diaphysis: their epidemiology, management and outcome. J R Coll Surg Edinb 42: 334
12. Weckbach A, Blattert TR (2001) Verfahrenswahl bei proximalen Unterschenkelfrakturen. Trauma Berufskrankh 3 [Suppl 2]: S143-S149

13. Wenzl ME (2004) Untersuchungen zur Leistungsfähigkeit eines multidirektional winkelstabilen Plattenfixateur interne Systems. Habilitationsschrift, Universität Lübeck

14. Wolter D, Fuchs S, Kranz HW et al. (2001) Tifanfixateur interne für die Tibia. Trauma Berufskrankh 3 [Suppl 2]: S156-S161

15. Wolter D, Jürgens Ch, Wenzl M et al. (2001) Titanfixateur-interne-Systeme mit multidirektionaler winkelstabiler Schraubenlage. Trauma Berufskrankh 3 [Suppl 4]: S425-S428

16. Wolter D, Schümann U, Seide K (1999) Universeller Titanfixateur interne - Entwicklungsgeschichte, Prinzip, Mechanik, Implantatgestaltung und operativer Einsatz. Trauma Berufskrankh 1: 307-319 